\title{
Semiclassical calculation of heavy-ion scattering in the chaotic regime
}

\author{
C. H. Dasso, ${ }^{1}$ M. I. Gallardo, ${ }^{1}$ and M. Saraceno ${ }^{2,3}$ \\ ${ }^{1}$ Departamento de Física Atómica, Molecular y Nuclear, Universidad de Sevilla, Apdo. 1065, E-41080 Sevilla \\ ${ }^{2}$ Departamento de Física, Comisión Nacional de Energía Atómica, Av. del Libertador 8250, Buenos Aires, Argentina \\ ${ }^{3}$ Escuela de Ciencia y Tecnologia, Universidad Nacional de San Martin, Alem 3901(B1653HIM), Villa Ballester, Argentina
}

(Received 20 September 2006; published 18 May 2007)

\begin{abstract}
The semiclassical approach has proven to be a most valuable tool for the construction of the scattering matrix and accurate evaluation of cross sections in a large variety of heavy-ion collision problems. In its familiar implementation, however, its use is restricted to what is now known as the "regular regime", as it makes use of classical reaction functions that must be continuous and interpolable. In this paper we identify what version of the semiclassical formalisms may be especially suitable for extension into the chaotic regime that develops at energies close to the Coulomb barrier. We also show the crucial role of the absorptive part of the ion-ion potential to retain the usefulness of the semiclassical methods under conditions of irregularity.
\end{abstract}

DOI: 10.1103/PhysRevC.75.054611

PACS number(s): 25.70.-z, 21.10.Re, 24.10.-i, 24.60.Lz

\section{INTRODUCTION}

The way to effectively compute scattering quantities within the semiclassical approximation in the regular regime has been known for many years. The semiclassical $S$-matrix construction of Miller [1] achieves the goal of expressing the $S$-matrix elements purely in terms of classical scattering trajectories with given boundary conditions. The main quantum mechanical effect incorporated in this kind of calculations is the interference between different trajectories and this simple feature allows accurate calculations of many reactions in the atomic, molecular [1] and nuclear [2] realms. In the regular regime the only issue is the validity of the semiclassical approximation itself and the accurate numerical calculation of classical trajectories. When the scattering is chaotic - an almost inevitable occurrence if a reaction barrier is present-many new features arise. From the numerical point of view, however, the main difficulty lies in the fact that a very large number of scattering trajectories can be found to fulfill the boundary conditions specifying a given reaction channel and it is not at all straightforward to expect that their interference will result in the reliable computation of observables.

In this paper we apply Miller's method to the semiclassical calculation of the scattering of heavy ions when the radial motion is coupled to a vibrational shape degree of freedom. We have previously explored $[3,4]$ several aspects of this problem, both classical and quantum mechanical, in the context of a simple model that sets correctly the nuclear sizes and interaction scales.

This paper is organized as follows. In Sec. II we recall the model that was used in Refs. [3,4] and in Sec. III we provide an overview of its classical characteristics. The semiclassical procedures are presented in Sec. IV, where we utilize the initial value representation (IVR) [5] to compute the $S$-matrix stressing the role of the absorption as a regularizing factor in the chaotic regime. A summary and conclusions close the paper in Sec. V.

\section{THE MODEL}

The relative motion of two heavy ions is described in first approximation by a potential model whose more relevant feature is the barrier created by the balance between Coulomb and centrifugal repulsion and the nuclear attraction. When shape degrees of freedom of the nuclear surface are considered, the relative motion interacts with their dynamics and the scattering is then described by a set of coupled equations. The deformations of the nuclear shape are described by the macroscopic variables $\alpha_{\lambda \mu}$ which characterize deviations from the equilibrium surface according to

$$
R(\theta, \varphi)=R_{\circ}\left[1+\sum_{\lambda \mu} \alpha_{\lambda \mu} Y_{\lambda \mu}^{*}(\theta, \varphi)\right] .
$$

The intrinsic degrees of freedom associated with these variables can be significantly excited in peripheral collisions [6]. Consequently, they play an important role in the modulation of the ion-ion interaction that affects barrier processes, such as those leading to fusion [7].

As a simple model we consider the coupling of the relative motion of two ions to an intrinsic harmonic mode, expressed by an effective Hamiltonian of the form

$$
H^{(\ell)}(r, p, \alpha, \Pi)=H_{\text {rel }}^{(\ell)}(r, p)+H_{\text {int }}(\alpha, \Pi)+V_{\text {coup }}(\alpha, r) .
$$

Here $r$ is the distance between the centers of mass of the colliding systems and $\alpha$ is a dimensionless variable that measures the (small) amplitude of the vibrational motion. The variables $p, \Pi$ are, respectively, their conjugate momenta. We take

$$
\begin{aligned}
H_{\mathrm{rel}}^{(\ell)}(r, p) & =\frac{p^{2}}{2 m}+\frac{\ell(\ell+1) \hbar^{2}}{2 m r^{2}}+U(r), \\
H_{\mathrm{int}}(\alpha, \Pi) & =\frac{C \alpha^{2}}{2}+\frac{\Pi^{2}}{2 D},
\end{aligned}
$$


where $m$ is the reduced mass, $\ell$ is the angular momentum and $C$ and $D$ the restoring force and mass parameters of the collective vibration. These last two quantities are related to the energy $\hbar \omega$ and deformation parameter $\beta$ of the mode by

$$
C=\frac{\hbar \omega}{2 \beta^{2}}, \quad D=\frac{\hbar}{2 \omega \beta^{2}} .
$$

The real potential $U(r)$ represents the combined effects of the nuclear and Coulomb interactions. For concreteness the nuclear potential is taken as a Woods-Saxon version of the Christensen-Winther empirical potential of [8]. The Coulomb potential is screened at large distances. The term that couples the intrinsic and relative motion variables, $V_{\text {coup }}$, arises from the Coulomb and surface-surface nuclear interactions between projectile and target. In leading order both these contributions are proportional to the deformation amplitudes. If a low multipolarity $\lambda$ for the mode is specified, it is possible within the present scheme to introduce an effective Coulomb form factor that reproduces a more appropriate radial dependence,

$$
V_{\text {coup }}(r, \alpha) \approx\left[-R_{\circ} \frac{\partial V_{N}}{\partial r}+\frac{3 Z_{1} Z_{2} e^{2}}{(2 \lambda+1)} \frac{R_{\circ}^{\lambda}}{r^{\lambda+1}}\right] \alpha .
$$

Note that the different $\mu$-components of a mode of multipolarity $\lambda$ have been combined in an effective "monopole" amplitude. The actual multipolarity of the mode is therefore taken into account through the radial dependence of the Coulomb component of the form factor. This procedure, justified in a coupled-channels approach because of their degeneracy in energy, is also suitable for the head-on case we treat below. However the main justification for these considerations is to make possible a realistic classical analysis that is not encumbered by the need to display many degrees of freedom.

When the nuclear densities overlap significantly a large number of channels open up that remove flux from the degrees of freedom treated explicitly. This is routinely treated in quantal calculations by adding an imaginary part to the ion-ion potential. We use here an absorptive potential of Woods-Saxon type

$$
W(r)=-\frac{W_{0}}{1+\exp \frac{r-R_{W}}{a_{W}}} .
$$

The parameters $R_{W}=4 \mathrm{fm}$ and $a_{W}=1 \mathrm{fm}$ have been chosen so that the absorption acts mostly inside the potential pockets but their values are otherwise not critical. With this fixed geometry the strength of the absorption will be adjusted only by the selected values of $W_{0}$.

\section{OVERVIEW OF CLASSICAL FEATURES}

The classical scattering trajectories are better described in the action-angle variables of the harmonic mode. They are related to $\alpha$, П by

$$
\begin{aligned}
& \alpha=\sqrt{(2 n+1) \hbar \omega C^{-1}} \cos \phi, \\
& \Pi=\sqrt{(2 n+1) \hbar \omega D} \sin \phi .
\end{aligned}
$$

At this point $n$ is a continuous variable, which will eventually become discretized at integer values in the quantum treatment.

Incoming trajectories are determined at $r_{i}=\infty$ by the initial relative momentum $p_{i}=-\sqrt{2 m\left(E-\left(n_{i}+\frac{1}{2}\right) \hbar \omega\right)}$ and by the oscillator variables $\phi_{i}, n_{i}$. Thus at constant $E$ the scattering is characterized by the classical map $n_{i}, \phi_{i} \rightarrow$ $n_{f}, \phi_{f} \cdot{ }^{1}$

Using this model we have studied [3] the chaotic layer that develops close to barrier energies and which brings drastic consequences in the organization of the classical motion. We summarize those results in Fig. 1, where we plot the reaction function $n_{f}\left(E_{\mathrm{tot}}, \phi_{i}\right)$ for a fixed $n_{i}=0$ at energies below and above the Coulomb barrier. The two pictures give an idea of the way in which the barrier region is disrupted by the coupling. In the uncoupled case the picture would be uniformly white $\left(n_{f}=n_{i}=0\right)$ and there would be a sharp straight line corresponding to the singular trajectory at the top of the barrier $(E \approx 56 \mathrm{MeV})$. This singularity reflects the fact that the unstable point at the top of the barrier is a bound motion and can only be reached asymptotically from the scattering region. The important fact is that this singular trajectory sharply

\footnotetext{
${ }^{1}$ To remove the free rotation of the angle $\phi$ with frequency $\omega$ a different variable $\phi \rightarrow \phi-\omega t$ is used so that $\phi_{i}, \phi_{f}$ are constant in the asymptotic region.
}
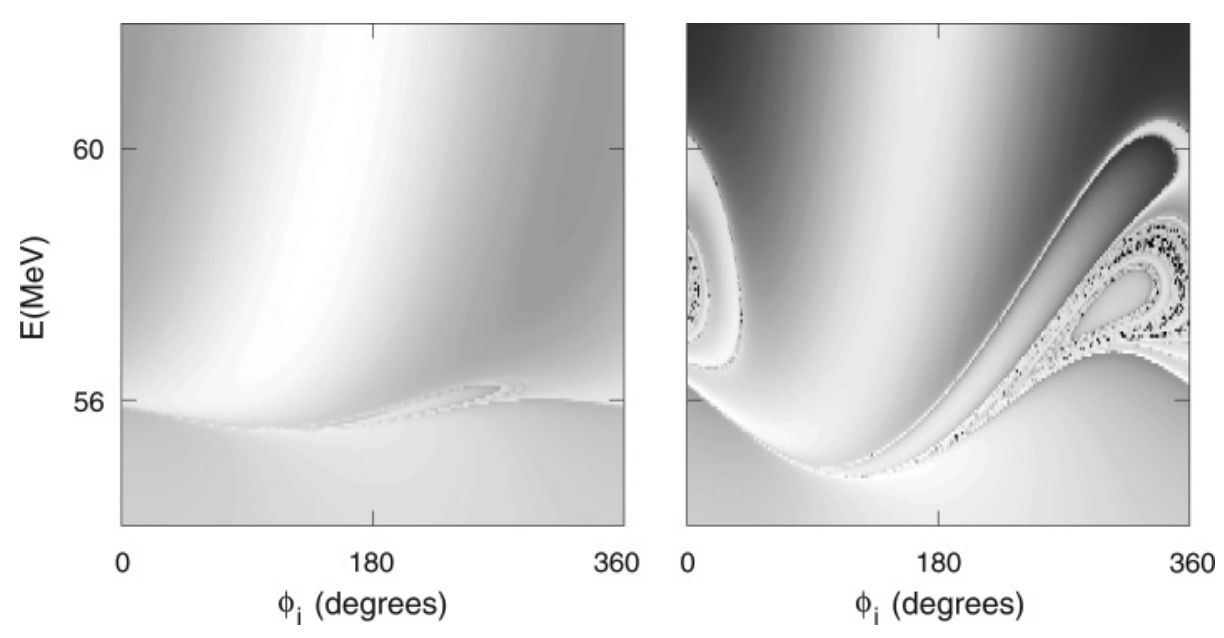

FIG. 1. Overall structure of the scattering map. The shades of gray give the value of $n_{f}$ (white for $n_{f}=0$, black for $n_{f} \approx 8$ ) for $n_{i}=0$. The left plot corresponds to $\beta=0.01$ and the right one to $\beta=0.04$. 
separates the classical motion that occurs above and below the barrier. Small values of the coupling $(\beta=0.01)$ bring about the possibility of excitation $\left(n_{f} \neq n_{i}\right)$ along with a mostly smooth dependence on $\phi_{i}$. The singular region is slightly distorted but still provides a clear separation for trajectories that scatter below or above the barrier. Larger values of the coupling $(\beta=0.04)$ start to display the full complexity of the barrier region. The "barrier" now extends over several $\mathrm{MeV}$ and is characterized by regions of smooth dependence separated by singular regions with structure on multiple scales. Well above and well below this chaotic layer the reaction functions are smooth and the trajectories have but one turning point either outside or inside the potential pocket. Within the irregular layer one finds smooth islands characterized by trajectories with more turning points and of increasing complexity which are responsible for the resulting fractal structures in the reaction functions.

\section{SEMICLASSICAL APPROXIMATION}

The classical scattering map has as an exact quantization the unitary $S$-matrix $\left\langle n_{f}|S(E)| n_{i}\right\rangle$ that yields the amplitude for populating the oscillator state $n_{f}$, when the incoming projectile is prepared in the state $n_{i}$. Both states are now labeled by integers $n_{i}, n_{f}$. The calculation of the quantal $S$-matrix is done numerically by means of coupled channel calculations that we have performed in [4]. These calculations allow us to compare with the semiclassical results and to judge the special difficulties encountered in the chaotic region.

An expression for the semiclassical $S$-matrix was given by Miller [1]

$$
\left\langle n_{f}|S(E)| n_{i}\right\rangle_{s c}=\sum_{\gamma}\left(2 \pi \mathrm{i} \frac{\partial n_{f}}{\partial \phi_{i}}\right)^{\frac{1}{2}} \exp \left(\mathrm{i} \Phi_{\gamma}\left(n_{f}, n_{i}\right)\right),
$$

where

$$
\Phi_{\gamma}\left(n_{f}, n_{i}\right)=-v_{\gamma} \frac{\pi}{2}-\int_{\gamma}\left(\frac{r d p}{\hbar}+\phi d n\right)
$$

and $v_{\gamma}$ is the additional Maslov integer that takes into account the points where the prefactor amplitude diverges. The sum involves all trajectories $\gamma$ that satisfy Hamilton's equations with the boundary conditions appropriate to the reaction channels: total energy $E$, initial and final values $n_{i}, n_{f}$ of the action variable for the oscillator. All quantities can be calculated for each scattering trajectory entirely in terms of classical mechanics. These quantities, although not routinely calculated in classical reaction codes, are easily incorporated along with the integration of trajectories.

This approach has two important drawbacks when one wants to apply it to irregular scattering. One is the search of trajectories that satisfy boundary — and not initial—conditions, and the second is that it diverges at the classical rainbows, and cannot provide reliable values in the classically forbidden regions. In the regular regime there are only a few (mostly two) independent trajectories contributing, the numerical search is quite feasible and the method has yielded precise estimates of excitation probabilities for rotational bands [9-11].
It is now clear what is the essential additional difficulty that the presence of chaotic behavior represents for this type of calculations. The very erratic nature of the reaction function means that the number of classical trajectories that satisfy the boundary conditions $n_{f}, n_{i}$ is not well defined (it will increase as we increase the number of trajectories per interval of $\phi_{i}$ ). The search for these trajectories is increasingly delicate numerically and the presence of many rainbows will make this calculation inaccurate at many points. More crucially the sum in Eq. (4.1) will have to take into account the interference of many trajectories with widely different actions. Questions about the convergence and reliability of this procedure are very severe. From the practical side it is certainly not very economical to have to compute and add an exponentially increasing number of scattering trajectories to come up with a number that is at best an approximation to the quantum result. This state of affairs is quite similar to what happens for the computation of energy levels from periodic orbits [12] where an exponentially increasing number of periodic trajectories have to be added to obtain a reasonable description of the spectrum of bound systems in terms of classical mechanics.

Another semiclassical approach that mitigates some of these problems is the initial value representation of the $S$-matrix $[1,5]$ :

$$
\left\langle n_{f}|S(E)| n_{i}\right\rangle=\frac{1}{2 \pi} \int_{0}^{2 \pi} d \phi_{i}\left(\frac{\partial \phi_{f}}{\partial \phi_{i}}\right)^{\frac{1}{2}} \exp \left(i F\left(\phi_{i}, n_{i}\right)\right),
$$

where $F\left(\phi_{i}, n_{i}\right)$ is a new phase function

$$
\begin{aligned}
F\left(\phi_{i}, n_{i}\right)= & -\int\left(\phi \dot{n}+\frac{r \dot{p}}{\hbar}\right) d t+\phi_{f}\left(\phi_{i}, n_{i}\right) \\
& \times\left[n_{f}\left(\phi_{i}, n_{i}\right)-n_{f}\right]-\mu \frac{\pi}{2}
\end{aligned}
$$

and $\mu$ another topological integer index associated with the trajectory. The phase $F\left(\phi_{i}, n_{i}\right)$ can be readily calculated from a classical integration of Hamilton's equations.

The integral in Eq. (4.3) can be performed analytically by stationary phase provided the saddle points are sufficiently separated, in which case each point contributes one term in the sum in Eq. (4.1). Close to the rainbows, two saddle points coalesce and the integral yields an Airy correction to the divergent prefactor, thus providing reliable amplitudes also in the forbidden region. If, on the other hand, the integral is calculated numerically by a uniform sampling of the interval $\phi_{i}=0, \ldots, 2 \pi$ with many trajectories the Airy pattern emerges automatically. The fundamental advantage of the IVR is then that the search problem has been avoided, the divergence at the rainbows has been regularized in exchange of a uniform sampling of the initial unobservable angle $\phi_{i}$. However, the essential difficulty arising from the addition of a large number of interfering trajectories subsists unabated.

We show in Fig. 2 the differences between the two semiclassical methods, as they apply to this model in the regular region, and how they compare with the exact coupled-channel results. The IVR was obtained sampling $\phi_{i}$ with $\approx 2000$ trajectories. As expected, the only significant differences occur near the edges 


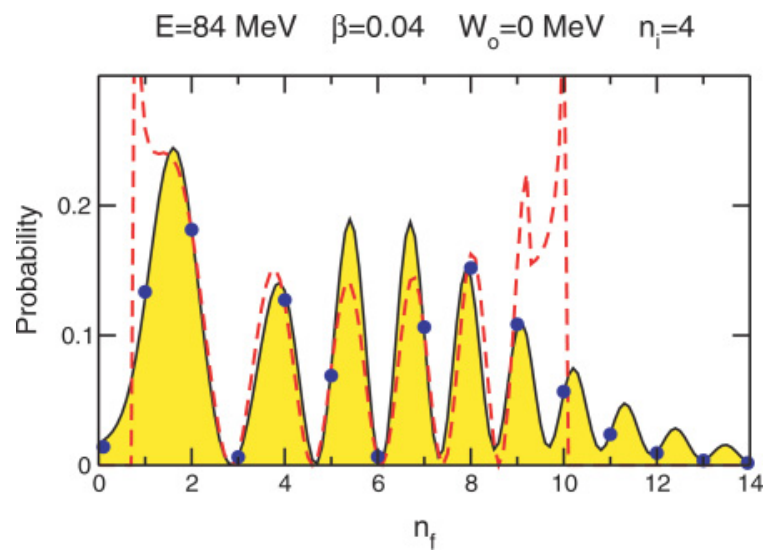

FIG. 2. (Color online) Comparison of the two semiclassical approximations with the exact results in the regular region. The shaded area is the semiclassical result using the IVR. The two Miller's $S$-matrix computed as a continuous function of $n_{f}$ to show more clearly the resulting interference pattern. At integer values it can be compared to the quantum results (heavy dots).

of the allowed region and the IVR is almost indistinguishable from the exact result, even in the forbidden region.

The IVR method has yielded excellent results in calculations in atomic and molecular physics and interest in it has revived in recent years in connection to its application to complex molecular structures. For a recent review of the method and its applications see [5]. This is then the method we propose for calculations in the chaotic regime. To sample the extreme variety of trajectories we estimate the integral in Eq. (4.3) by discretizing $\phi_{i}$ and propagating up to 20000 trajectories. Figure 3 illustrates the advantages and the difficulties of this semiclassical procedure.
It shows results in the regular and the chaotic regimes at $E=$ $84 \mathrm{MeV}$ and at $E=74.5 \mathrm{MeV}$ with $\beta=0.04$. The top part shows how the initial set of conditions $n_{i}=4, \phi_{i}=$ $0, \ldots, 2 \pi$ is mapped by the scattering process into the curve $n_{f}, \phi_{f}$. (Notice that, just as it was also done in Refs. [3,4], unrealistic boundary conditions have been chosen to improve the pedagogical value of the illustration.) The bottom part gives the IVR result for the excitation probabilities together with the quantum results from a coupled-channel calculation. The purely classical result obtained by simply adding probabilities is also shown. In this part of the calculation there is no absorption $\left(W_{0}=0\right)$ and therefore the $S$-matrix is unitary within the populated channels and in the classical and semiclassical calculations all trajectories contribute uniformly.

In the regular case the scattering occurs well above the barrier and the trajectories have a single turning point. The results can be well understood in terms of the interference of just two trajectories which produce the interference pattern superimposed on the classical result. The Airy pattern arising from the interferences near the rainbows is clearly visible. The agreement with the results of a coupled channel calculation is systematically excellent.

In the chaotic case at $E=74.5 \mathrm{MeV}$ the situation is radically different. The IVR result is essentially random and no agreement can be discerned with the exact results. The reason appears as soon as one looks at the classical organization of the scattering trajectories: each scattering channel is fed by a large amount of trajectories all with different phases and amplitudes. Because of the finite sampling of initial trajectories it is impossible to describe well the complicated interference pattern.

To circumvent these difficulties we take advantage of the fact that no realistic ion-ion potential is purely real. The
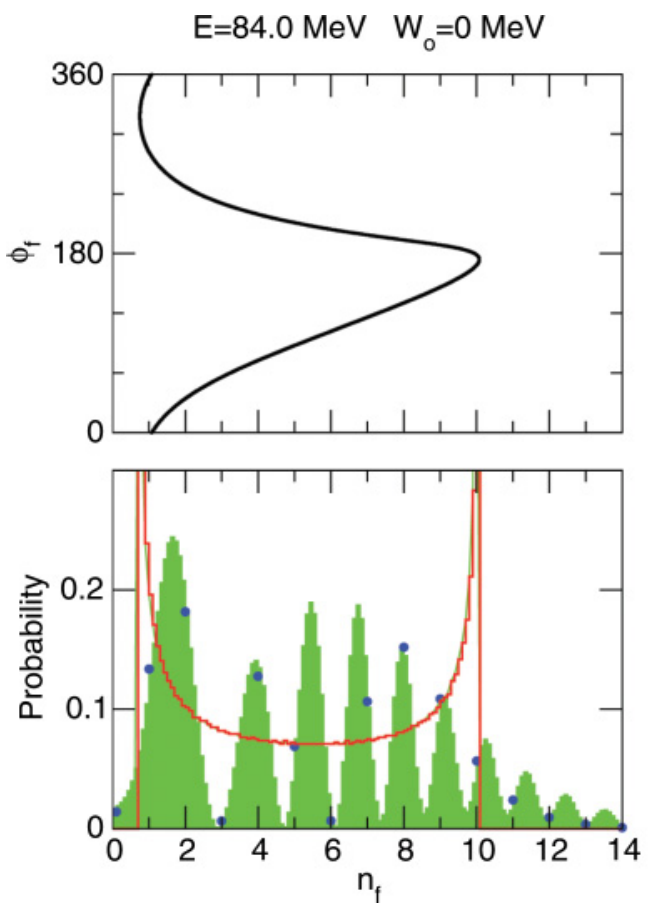
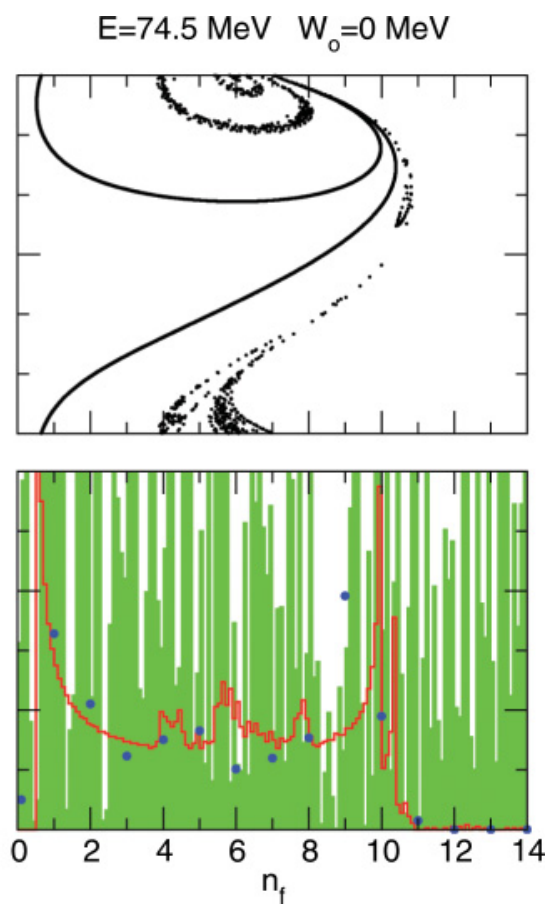

FIG. 3. (Color online) Semiclassical calculation of the excitation probabilities for the different reaction channels in the absence of absorption, $W=$ $0 \mathrm{MeV}$. The reaction considered is ${ }^{40} \mathrm{Ca}+{ }^{40} \mathrm{Ca}$ and the initial state of the harmonic mode with $\hbar \omega=2 \mathrm{MeV}$ and $\beta=0.04$ is specified by $n_{i}=4$. The two frames to the left correspond to a total energy of $E=82.0 \mathrm{MeV}$ (regular regime) while the two frames to the right are for $E=74.5 \mathrm{MeV}$ (chaotic regime). In each column the top frame shows the initial values $\phi_{i}[0,2 \pi], n_{i}=4$ mapped to the final ones $\phi_{f}, n_{f}$. The lower frames show the classical distribution of probability (unshaded histogram) and the IVR result (shaded histogram). The dots give the quantal results from a full coupled-channel calculation. 

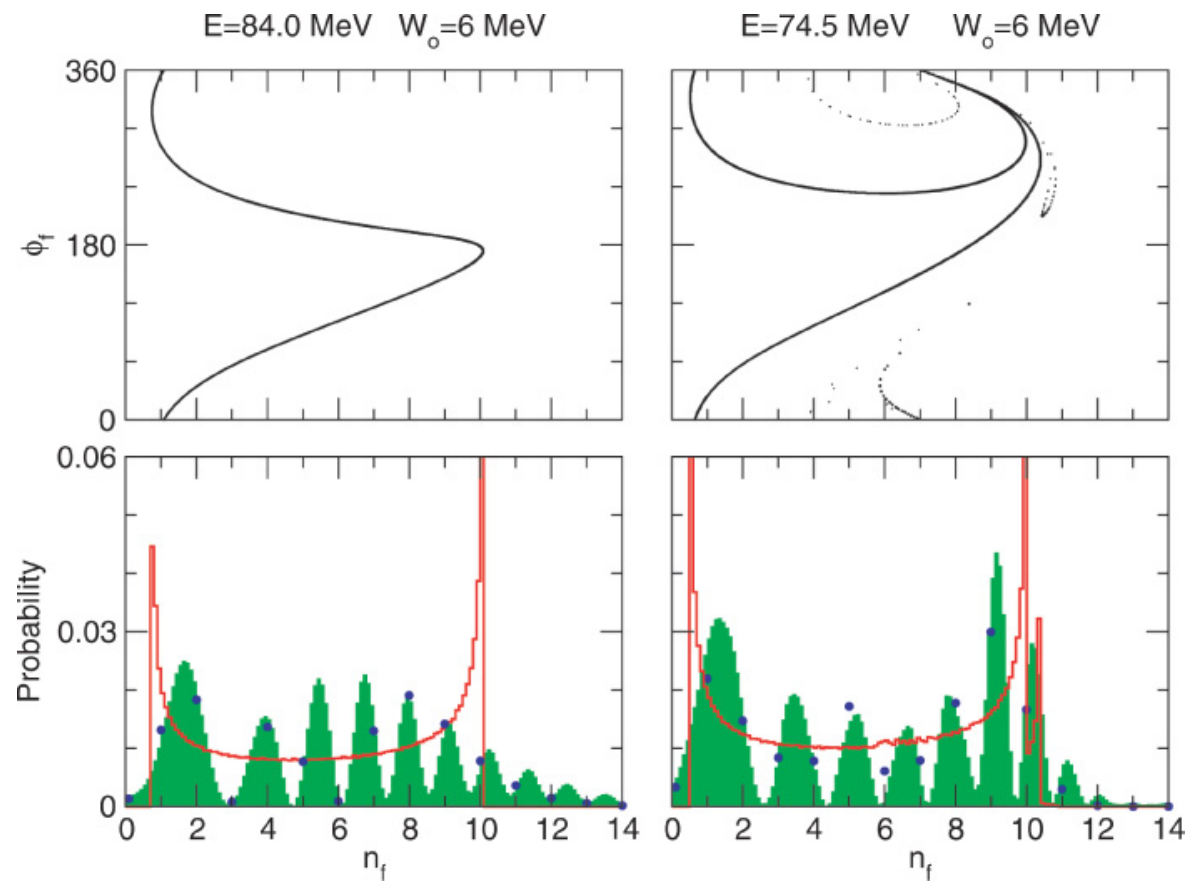

FIG. 4. (Color online) Semiclassical calculation of the excitation probabilities for the different reaction channels in the presence of absorption. This figure is analogous to Fig. 3, except for the value of $W_{0}=6 \mathrm{MeV}$. The effect of the absorption is represented by drawing each point with an area proportional to its survival probability $\rho_{\gamma}$. complex optical potential is readily incorporated into standard heavy-ion coupled channel reaction codes and results in non unitary scattering matrices. Classically — and in leading order in $W_{0}$-it is customary to incorporate the effects of absorption by assigning to each classical trajectory a survival probability

$$
\rho_{\gamma}=\exp \left[\frac{2}{\hbar} \int_{-\infty}^{\infty} W\left(r_{\gamma}(t)\right) d t\right],
$$

where $W(r)$ is the imaginary potential in Eq. (2.7). Changing the value of $W_{0}$ results in a selective removal of the more complicated trajectories that have long dwell times in the interaction region. In fact, the role of $W_{0}$ is quite analogous to that of a small imaginary part in the energy for bound state calculations in the chaotic regime. It regularizes the periodic orbit sums at the expense of introducing an energy smoothing. Here it appears naturally as a result of a physical mechanism and its strength in a given situation is a measure of the observability of chaotic effects.

In Fig. 4 we show the same results as in Fig. 3 but now with an added absorptive potential with strength $W_{0}=6 \mathrm{MeV}$. We have translated its effect by drawing the point corresponding to each trajectory with an area proportional to the survival probability $\rho_{\gamma}$. In the regular case all trajectories are well above barrier and they all share a similar portion in the radial region where the absorption is active. Thus they are all damped essentially by the same factor. Therefore the whole interference pattern is simply scaled by this factor. This is clearly seen in the pattern of excitation probabilities, which is almost identical to that in Fig. 3, just reduced by a constant factor. The agreement with the quantum calculation, which now includes the imaginary part of the optical potential, remains excellent.

Absorption acts in a very different way in the chaotic case. The trajectories that feed one given channel are now of widely different nature, the simpler just exploring the interaction region once, while the more complicated can have many turning points and spend long times in it. They are therefore damped with very different factors, thus changing substantially the resulting amplitude. We clearly observe this fact in the top right part of Fig. 4 where the complicated trajectories are now absent. As the strength of $W(r)$ increases, out of the many trajectories feeding one given channel and interfering with almost random patterns only the simplest survive the damping. The interference is mainly produced by two simple branches but some channels are still fed by the remaining irregular trajectories. The resulting patterns are now in good agreement with the coupled-channel calculation.

\section{CONCLUSIONS}

The lesson to be learned from this exercise is twofold. From the point of view of the semiclassical computability of chaotic scattering we conclude that the straightforward superposition of scattering trajectories, even with the improvements introduced by the IVR, cannot yield even semiquantitative results in the chaotic regime. Whether more sophisticated techniques involving resummations [13] can yield better results in this case remains an open challenge, but probably of very limited practical use for realistic nuclear reactions. On the other hand we have shown that absorption-strongly present in nuclear heavy ion reactions - provides a natural way of selectively damping the more irregular trajectories, thus regularizing the scattering trajectory sum and restoring the feasibility of semiclassical calculations. One could argue that strictly speaking the most severe aspects of chaos have been eliminated by introducing absorption, and that what remains is a finite collection of scattering trajectories which will be more or less complicated according to the strength of the imaginary potential. We think that this is indeed the right (and practical) 
way to think about this issue in the context of nuclear reactions, and that the presence of special "transparency windows" [14] in some selected reactions provide opportunities to observe it [15-17].

[1] W. H. Miller, J. Chem. Phys. 53, 3578 (1970).

[2] R. Broglia and A. Winther, Heavy Ion Reactions (AddisonWesley Pub. Co., Redwood City CA, 1991).

[3] C. H. Dasso, M. Gallardo, and M. Saraceno, Nucl. Phys. A549, 265 (1992).

[4] C. H. Dasso, M. Gallardo, and M. Saraceno, Nucl. Phys. A587, 339 (1995).

[5] W. H. Miller, J. Phys. Chem. A 105, 2942 (2001).

[6] S. Landowne and A. Vitturi, in Treatise on Heavy-Ion Science, Vol. 1, edited by D. A. Bromley (Plenum, New York, 1984).

[7] M. Beckerman, Phys. Rep. C 129, 145 (1985).

[8] P. R. Christensen and A. Winther, Phys. Lett. B65, 19 (1978).

[9] A. B. Balantekin and S. Kuyucak, Fusion 97 [J. Phys. G: Nucl. Part. Phys. 23, 1159 (1997)]; M. Dasgupta, D. J. Hinde, N. Rowley, and A. M. Stefanini, Annu. Rev. Nucl. Part. Sci. 48, 401 (1998).

[10] H. Esbensen, Nucl. Phys. A352, 147 (1981).

\section{ACKNOWLEDGMENTS}

We acknowledge support from the Ministry of Education and Science under project nos. FIS2005-01105, FPA200504460, from ANPCyT-PICT25373 and Conicet-PIP6137-04.

[11] C. H. Dasso, S. Landowne, and A. Winther, Nucl. Phys. A405, 381 (1983)

[12] Chaos 2, 1 (1992), special edition on Periodic Orbit Theory, edited by P. Cvitanović.

[13] B. Georgeot and R. E. Prange, Phys. Rev. Lett. 74, 4110 (1995).

[14] C. H. Dasso, M. Gallardo, and M. Saraceno, Phys. Rev. Lett. 77, 3747 (1996).

[15] C. H. Dasso, G. Pollarolo, and M. Saraceno, Nucl. Phys. A602, 77 (1996).

[16] G. V. Martí, A. J. Pacheco, J. E. Testoni, D. Abriola, O. A. Capurro, D. E. Di Gregorio, J. O. Fernández Niello, E. Achterberg, and D. E. Álvarez, Phys. Lett. B447, 41 (1999).

[17] G. V. Martí, A. J. Pacheco, J. E. Testoni, D. Abriola, O. A. Capurro, D. E. DiGregorio, J. O. Fernández Niello, A. O. Machiavelli, R. M. Clark, P. Fallon, A. Goergen, D. Ward, C. Y. Wu, A. Hayes, D. Cline, and R. Teng, Braz. J. Phys. 34, 3A 885 (2004). 